\title{
Developing the Control System for a Novel Electric Medical Operation Table
}

\author{
Wei Luo \\ Department of Mechanical Engineering \\ East China University of Science and Technology \\ Shanghai 200237, China \\ Jun Xu \\ Department of Mechanical Engineering \\ East China University of Science and Technology \\ Shanghai 200237, China \\ Jin-Long Luo \\ Department of Mechanical Engineering \\ East China University of Science and Technology
}

\author{
Jian-Jun Yi \\ Department of Mechanical Engineering \\ East China University of Science and Technology \\ Shanghai 200237, China \\ Shao-Ru Zhang \\ Department of Mechanical Engineering \\ East China University of Science and Technology \\ Shanghai 200237, China \\ jjyi@ecust.edu.cn \\ Shanghai 200237, China
}

\begin{abstract}
At present, most of the operation table has single functions and own drawbacks like high cost, and difficult to repair after failure. In this context, a novel control system of the electric operation table is proposed in this paper. The control system mainly includes handheld controller and main control board. Hand-held controller is used to send commands to the main control board to perform the appropriate actions, such as setting the time, PWM value, compound position, and display the current action, battery power, fault information etc. Main control board manipulates the different attitude adjustment, angle detection, data exchange with the remote monitoring center, fault self-diagnosis, behavior recording, etc.. Through the validation, the developed control system was proved to meet the requirements and technical parameters. By EMC testing, it can run stably and match the related standard of EMC. The developing method, especially the remote fault diagnostics method proposed in this paper can be applied to the remote monitoring, maintenance, fault diagnosis of other mechatronic system.
\end{abstract}

Keywords-Medical Electrical Operation Table, Control System, EMC Standard

\section{INTRODUCTION}

As one of the basic medical equipment, operation table occupies a very important position in the orthopedic, orthopedic surgery and surgical medical devices products [1]. Therefore, domestic and foreign medical institutions has carried out extensive research, developed a variety of operation table devices [2]. However, a considerable large number of the operation tables in hospitals are not satisfactory. The main shortcomings are shown concretely as follows, the structure of the operation table control system is complex, performance is not stable enough, and high failure rate, etc.. Additionally, the cost level is generally high, in this context, developing a high-end, more stable and practical operation table is imperative [3].

A new type control system of electric operation table is required to carry out the multiple attitude motions, such as operation table's table-board up and down, inclines left, right, forward, and backward, the backplane adjusting motion, etc. The wired handheld controller is need to connect a screen for displaying operation actions and device status. Fault selfdiagnosis module is implemented to test the failure of each module. At the same time, with infrared control, wireless controlling of the operating is realized. Functions like reset, behavior record, remote monitoring and self-monitoring and diagnosis are also necessary to be developed in the operation table.

The objectives of this paper are to describe the methodologies of complex embedded control system, especially the remote fault diagnostics method proposed in this paper can be applied to the remote monitoring, maintenance, fault diagnosis of other mechatronic system.

The rest of this paper is organized as follows. System structure scheme of the operational table is presented in Section 2. The development and implementation of control system and controller design are presented in Section 3. The realization of the software is provided in Section 4, and the paper is concluded in Section 5.

\section{SYSTEM STRUCTURE}

The appearance and basic structure of the electric operating table is shown in Figure.1, it mainly includes tableboard, main body, electric control part, etc. There are twelve operating table's attitudes can be controlled, including the table-board up and down; inclines left, right, forward, and back; backplane fold up and down ; base translation and lock[4]. 


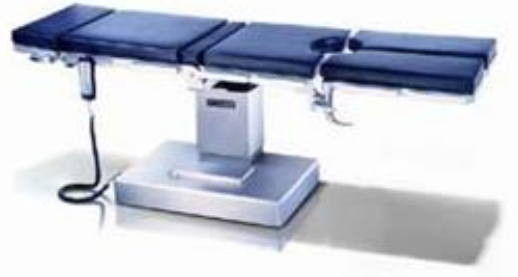

Figure 1. The appearance and basic structure of electric operation table

The structure chart of the electric operation table control system is shown in Figure.2. The control system includes wired handheld controller, infrared controller, three-axis acceleration sensor, main control board, power supply, drives, brushless DC motors, electromagnetic valve, hydraulic pumps, hydraulic cylinders, operation table and other parts. The electric operation table's control is realized through sending commands to the main control board by the controller. After the control board receives an operation instruction, switch of the electromagnetic valve and drive power supply is opened. Then the PWM signal is generated to control rotation of the brushless DC motor. Through the hydraulic pump, pressurized oil are transfered to the electromagnetic valve to control the movement of the hydraulic cylinder to the specify direction, and finally the operation table could react to the corresponding action. When operation table carried out an action, the sensor installed in it will be rotated with it, through the data from the sensor, the angle of the table could be achieved, thus can record the operating table's movement attitudes, and achieve function like one key reset function and various composite attitude adjustment.

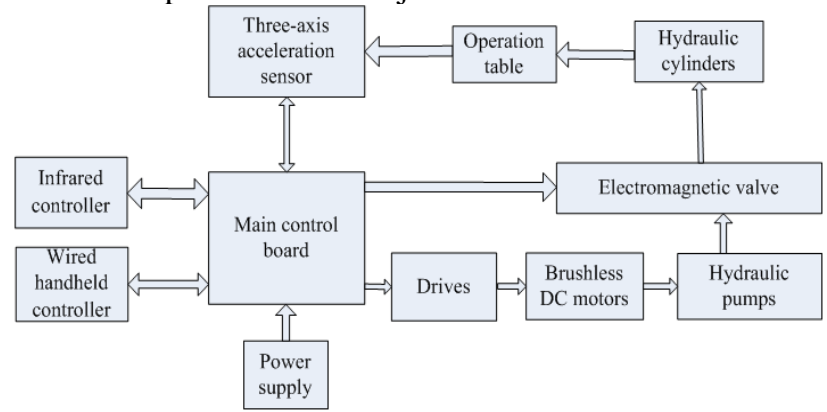

Figure 2. The structure chart of the electric operation table control system

\section{The DeVElopment AND IMPLEMENTATION OF CONTROL SYSTEM}

\section{A. Hand-held Controller}

Handheld controller is an important control part of the operation table. The electro-hydraulic control of operation table is completed according to the instructions sent from the hand press controller. Hand-held controller is consist of infrared controller and the wired handheld controller. Wired handheld controller communicates with the main control board by CAN BUS. The control pannel is consist of a total of 21 keys, including the power key, shutting down key, composite key and reset key, composite posture adjustment $\mathrm{key}$, four function keys and 12 action buttons. The composite key works as a master operation switch, to prevent misoperation, the composite key and action buttons should be pressed at the same time to give certain control actions. Function keys are used to set the PWM value, sensor calibration, and time or query the SD card allowance, fault detection, etc. At the top of the hand-held controller, there is a display screen, the state of operation and equipment can be show on it. The information on the screen includes current operating actions, power, and time and so on. In the figure. 3 is the picture of the wired controller. The function of the infrared controller and wired handheld controller are basically the same. The reason for using infrared control is that it is a wireless, non-contact control method, with the advantage of strong anti-interference ability, reliable information transmission, low power consumption, low cost, easy to implement, etc. And the infrared controller uses NEC protocol.

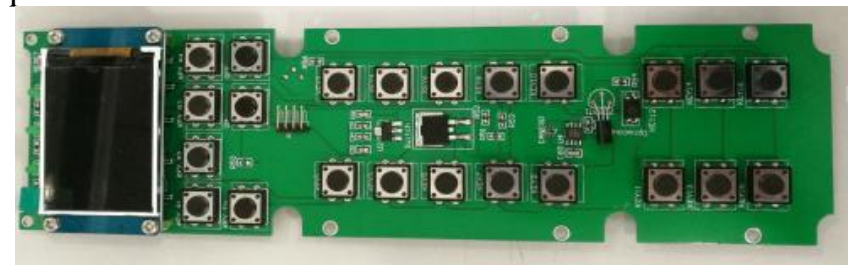

Figure 3. Wired Controller

\section{B. Sensor}

In order to realize functions like operation behavior record, reset and composite attitude adjustment, the system adopts a three-axis acceleration sensor ADXL345 to detect the angle incline, figure. 4 is the physical map of the sensor module.

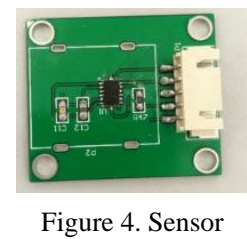

To realize this function, it requires to install two threeaxis acceleration sensors in the operation table, one in the table-board, sensor and table-board are parallel, which is used to measure the table-board left, right and forward, backward incline angle, as X and Y respectively; another sensor is in the backplane, used to measure the backplane fold up and down angle, set as X1. When resetting or adjusting the composite attitude, the center of the sensor chip is set as the coordinate origin, then set the X, Y, X1 axis in the positive direction, and the attitude could be adjusted in three directions individually. The design requirements to adjust the $\mathrm{X}$-axis direction attitude first, then adjust the $\mathrm{Y}$-axis direction attitude, finally adjust the $\mathrm{X} 1$-axis direction attitude. The stopping angle of three axial directions is set to $-0.5^{\circ}$ to $0.5^{\circ}$. When the angle is reached in a certain axis direction, the operation table stops moving. Figure. 5 is the operation table reset flow chart. The principle of composite posture adjustment principle is the same with the reset. 


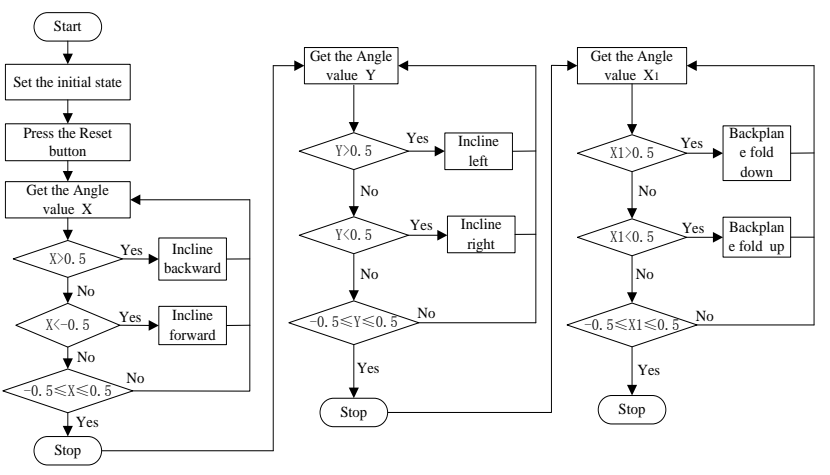

Figure 5. The operation table reset flow chart

\section{Main Control Board}

Main control board is the most important part of the whole control system. Shown in Figure. 6 is the structure chart of the control board, including the microprocessor module, power control module, drive and electromagnetic valve control circuit module, data storage module, fault selfdiagnosis module, WIFI module, etc. In the figure. 7 is the picture of the main control board.

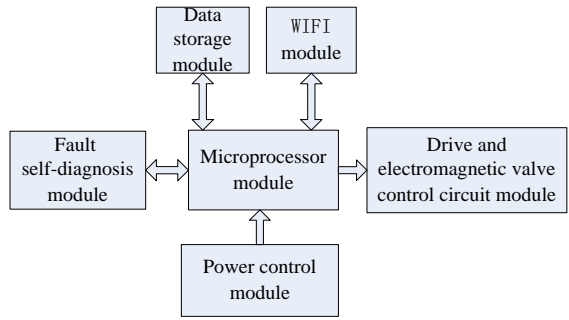

Figure 6 . The structure chart of the control board

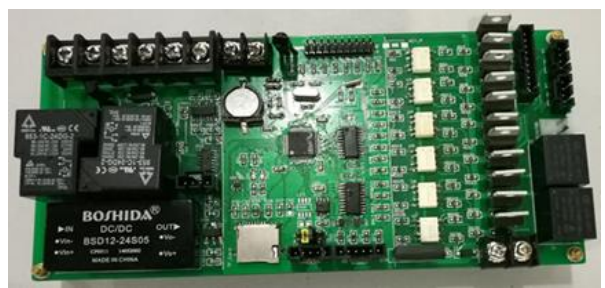

Figure 7. Main control board

The main task of the microprocessor module main tasks is: electromagnetic valve and motor control circuits module control, power supply control, motor speed control, sensor data processing, communication with handheld controller, control the SD card data storage to achieve the record of operating table's movement attitudes, control module fault self-diagnosis, WIFI module data transmission control, etc. Adopting microprocessor design can simplify the circuit design, reduce the line fault point, and ensure the reliability of the action[5].

The power control module is mainly consist of relay, reverse Schmidt trigger, voltage comparison chip, $24 \mathrm{~V}$ to $5 \mathrm{~V}$ module, etc. In order to prevent accidental interference which could lead to operation table occur action, only when the action button is pressed, the motor and the electromagnetic valve are powered on. When power is supplied by an external power, the module controls the external power to charge the battery at the same time. When the battery is charged, the battery is disconnected and protected. When there is no external power supply, the battery is used for power supply.

Driver and electromagnetic valve control circuit module is mainly responsible for completing the control of the driver and electromagnetic valve control. When controlling the movement of the operation table, the driver is supplied with a PWM signal to control the rotation of the brushless DC motor, and control the opening and closing of the electromagnetic valve switch to make the pressure oil flow in the specified direction. There is also strong and weak electric isolation function, to prevent the interference of motor to the minimum system or other modules.

Data storage module uses a SD card to store data, after the microprocessor reads the angle data of the ADXL345 which detects the tilt of the operating table, the data is stored in the SD card, which could record the current time and specific action of the operation. If there is a misoperation in a surgery, which caused the accident, the record can be read from the main control board SD card and handheld controller SD card, which could be used for accident tracking. If someone operates the handheld controller and presses the action button at the same time, then this is a human error accident. If there is no record of the buttons operation at this time, then this accident is not caused by man, it is the problem of operation table products or the result of interference.

Fault self-diagnosis module is used to test the fault condition of each module. It includes boot self-diagnosis and menu bar self-diagnosis. Boot self-diagnosis mainly checks the CAN communication and SD card failure automatically. If there is a fault, display it on the screen to remind after boot, if no, do not show. The menu bar self-diagnosis is manually, when the equipment trouble happened, user can enter the selfdiagnosis function from the menu bar, after pressing the button, the main control board will check the main modules, and the failure information of the fault module will be displayed on the screen. The system can realize the selfdiagnosis include electromagnetic valve, motor, infrared receiving head, three-axis acceleration sensors, SD card module.

WIFI module mainly interacts data with remote monitoring center. During surgery, the power value, current attitude, incline angle and other state parameters are sent to the monitoring center, which could facilitate remote selfdiagnosis and maintenance.

\section{The Realization of the Software}

The design of the software uses structured programming method, every module is independent of each other, to facilitate post code maintenance, migration, upgrading and debugging[6].Through the analysis of main control board function, the software design is divided into three main modules, including action control program, query program, set up program. And the detailed design program of each module is divided into several small modules, as shown in figure. 8 . 


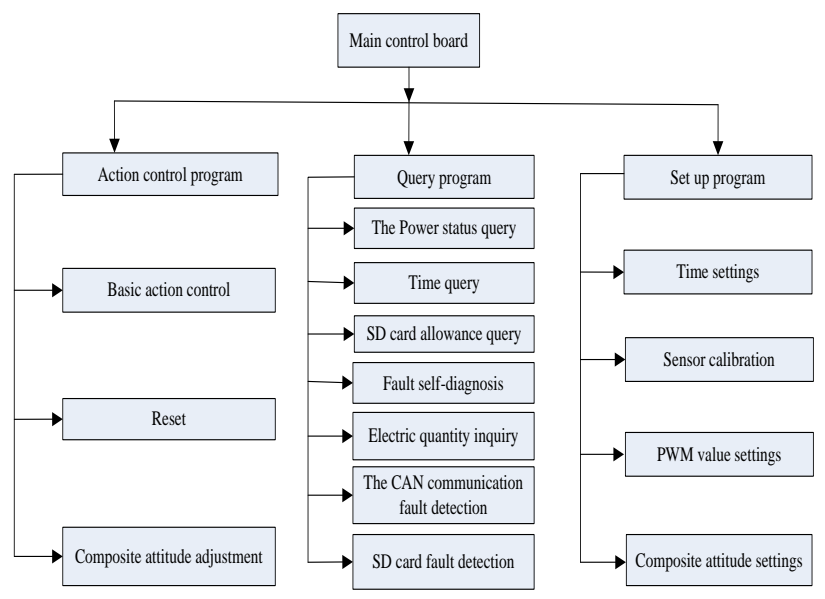

Figure 8 . The system software function module

Main control board action control program mainly includes the electromagnetic valve and motor control circuit, reset, composite attitude adjustment, table-board and backplane angle detection module program, etc. As shown in figure.9, first is the initialization of the system clock, IO port, sensors, SD card, etc. And then the main program will obtain the key values sent by the handheld controller. Then according to the key commands, system will perform the corresponding action, detect the angle at the same time, functions like reset and composite attitude adjustment, and record the operation behavior in SD card could also work properly.

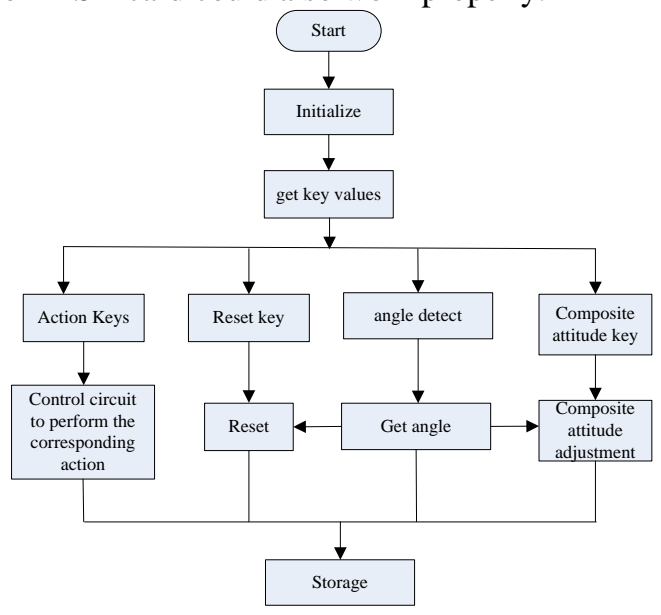

Figure 9. Motion control program flow chart

In this paper, a single operation table control system is designed. In order to facilitate management and reduce the failure probability of the operation table control system during surgery, according to the application requirements, a remote online monitoring method is developed as shown in figure.10. Operation table will be numbered and exchange data via the built-in WIFI module with remote monitoring center. Then the system can realize on-line monitoring of the operation table posture transformation, battery level, tilt angle and other state parameters. According to the data collected by the sensors and information from the main control board, regular remote fault detection could be realized to detect and analysis the fault of the operation bed and give considerable solutions. The remote online monitoring center could also support the system remote upgrade which makes maintenance more convenient.

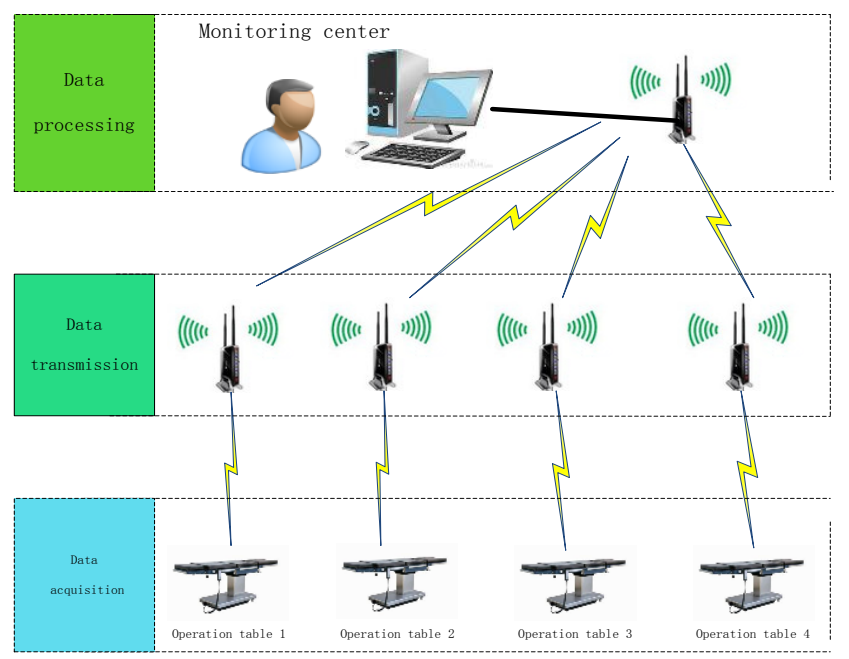

Figure 10. Electric operation table monitoring management system

\section{CONCLUSION}

After testing, the whole control system of operation table runs stably. The following functions are realized in this operation table, which include the basic motion control, reset, composite attitude adjustment, operation behavior records, accident tracking, infrared control, system fault selfdiagnosis, remote monitoring and maintenance, charging protection, automatic power off, etc. The operation table developed in this paper could meet diversity functional requirements at low cost, and it is valuable in real application. The entire operation table electrical control parts compliance with electromagnetic compatibility design specifications, and passed the EMC test. The radiation and conduction test is also passed respectively, are shown in figure. 11 and figure. 12 below.

Finally, the remote online monitoring and fault diagnostics method proposed in this paper can be applied to the other mechatronic system, and has practical application value.

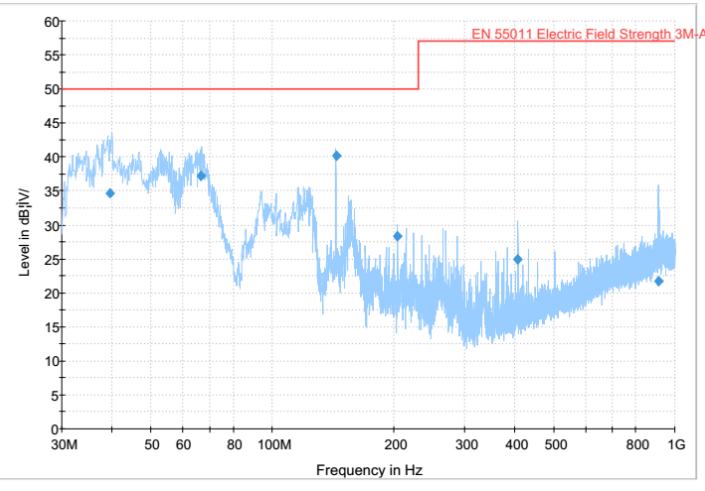

Figure 11. Radiation test 


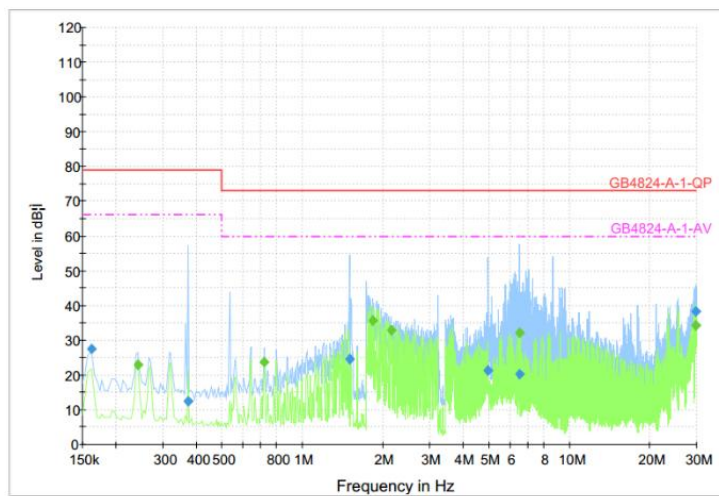

Figure 12. Conduction test

\section{ACKNOWLEDGMENTS}

This paper was supported by the Research Foundation of Science and Technology Commission of Shanghai under Grant No. 10DZ1500200, the Natural Science Fund of China
(NSFC) under Grant Nos. 50975088, 51275173, 51575186 and 51210105018, the Fundamental Research Funds for the Central Universities under Grant No. WH0913009, Shanghai Pujiang Program under Grant No. PJ201000353, and Shanghai Software and IC industry Development Special Fund under Grant No. 120493.

\section{REFERENCES}

[1] Zheng fu, Military medical equipment [M]. Beijing: People's Military Medical Publisher, 2004, pp.562-566.

[2] Xiao-Jun Sun, Study of key technology of portable operating table [D]. Tianjin University, 2008

[3] Chi guo, Yong-Wei Mi, Sui Zhang. Design and application of a reliable and power saving operating table control circuit $[\mathrm{J}]$. China Medical Devices, 2009, 24(06), pp.21-22.

[4] Wei yan Jiang, A new type of electric operation table controller [J]Electronic World 2014(14), pp.27-28.

[5] Yu xuan Wang, Application and supervision of electric operation table in clinic [Conference Paper] 2011.

[6] Qiang Meng. The data acquisition system design based on STM32 [D]. Nanjing Forestry University, 2014. 